\title{
The COVID-19 pandemic and nuclear cardiology: An opportunity to grow stronger?
}

\author{
Hicham Skali, MD, MSc, FACC ${ }^{\mathrm{a}, \mathrm{b}}$ \\ a Division of Cardiovascular Medicine, Department of Medicine, Brigham and Women's Hospital \\ and Harvard Medical School, Boston, MA \\ b Division of Nuclear Medicine and Molecular Imaging, Department of Radiology, Brigham and \\ Women's Hospital and Harvard Medical School, Boston, MA
}

Received Sep 9, 2020; accepted Sep 10, 2020

doi: $10.1007 / \mathrm{s} 12350-020-02383-y$

\section{See related article, pp. 331-335}

$$
\begin{array}{r}
\text { "In the middle of difficulty lies opportunity" } \\
\text { - Albert Einstein }
\end{array}
$$

On March 11th, 2020 the World Health Organization officially declared COVID-19 a pandemic. ${ }^{1}$ By then, the disease caused by SARS-CoV-2 had spread to 114 countries and affected 118,000 cases.

Given the exponential surge in the number of cases and constraints placed on hospital resources, national health authorities implemented total or partial lockdown procedures or stay-at-home orders or advisories. Healthcare systems, hospitals and practitioners canceled or postponed most (if not all) elective and non-urgent visits, tests and procedures at once. Only essential and urgent encounters were conducted. This unprecedented operational shift happened essentially over a brief period of time (overnight?). Several healthcare leaders were calling their colleagues elsewhere asking for help and sharing ideas. However, the circumstances were unique, unparalleled for everyone and healthcare professionals had to be creative and resourceful while mitigating the risk of infection spread, promoting safety for healthcare workers and patients, and following national and local orders and infection control recommendations.

\footnotetext{
Reprint requests: Hicham Skali, MD, MSc, FACC, Division of Cardiovascular Medicine, Department of Medicine, Brigham and Women's Hospital and Harvard Medical School, 75 Francis St, Boston, MA 02115; hskali@bwh.harvard.edu

J Nucl Cardiol 2021;28:336-7.

$1071-3581 / \$ 34.00$

Copyright (C) 2020 American Society of Nuclear Cardiology.
}

Italy, with Spain and France, was one of the most hardly hit countries in the beginning of the pandemic. By the end of April 2020, just 6 weeks into the pandemic, there had been 179,192 cases and 28,236 deaths. Italian doctors, nurses, technologists, and administrators were pulled into a battle to manage the thousands of daily new cases, and hundreds needing a hospital or ICU admission. At the same time, other non-COVID-19 conditions, including cardiovascular or oncologic, continued to present, though at a much lower rate ${ }^{2}$ or much later in the disease course.

In this issue of the journal, Scrima et al. provide an early account of their initial experience in establishing an ad hoc protocol that allowed them to continue providing nuclear cardiology services during the pandemic. This protocol required multi-disciplinary collaboration between clinicians, technologists and administrators. It was based on proper case selection and prioritization, detailed screening for symptoms and risky contacts on days before and day of the procedure, protocol modification to allow for shortened scans based on stress first, as well as universal face-masking and gloves, physical distancing and hand hygiene, and PPE for healthcare professionals.

In their experience, between April 7th and May 15 th, none of the 46 patients they studied reported any complications or COVID-19 symptoms assessed by telephone follow-up. None of the healthcare providers had positive serologic testing conducted on May 18th, 2020. Moreover, using a stratification strategy to prioritize cases seemed to identify a higher proportion of patients with significant inducible ischemia (19.4\%) compared to the same period in 2019 (7.7\%) without similar stratification and before COVID-19.

The authors need to be commended for their effort in developing a protocol to allow the continuous and safe operations of their nuclear cardiology laboratory 
during the peak of the pandemic in their country. They were able to provide a necessary clinical service to their patients and collect data about the feasibility and safety of the utilized protocol, while also battling a ravaging pandemic.

The American Society of Nuclear Cardiology (ASNC) was able to draw on its worldwide collaborators and their experiences to coordinate a successful webinar titled "COVID-19 Preparedness for Nuclear Cardiology Labs: Insights from the US, China and Singapore",3 on March 24th, 2020 with over 500 live attendees. The highlights from that webinar stressed the importance of appropriate screening and prioritization of procedures, separation of COVID and non-COVID patients, use of face mask, hand hygiene, and physical distancing for patients and healthcare workers. The precautions highlighted here were not novel but followed a simple outline that tracked a patient's journey through the nuclear cardiology laboratory.

These recommendations were also detailed in an information statement by ASNC and the Society of Nuclear Medicine and Molecular Imaging, available on a pre-print server as early as April 2nd, $2020^{4}$ before the final publication in both the Journal of Nuclear Cardiology $y^{5}$ and the Journal of Nuclear Medicine.

A month later, on April 30th 2020, as the number of new COVID-19 cases started to slow down, another successful webinar by ASNC was presented and emphasized key lessons to safely resume nuclear cardiology activity for non-emergent cases, ${ }^{6}$ and was also followed by another information statement by ASNC and SNMMI and endorsed by the Infectious Diseases Society of America. ${ }^{7}$ This document stressed the importance of following national and local recommendations based on local tracked cases, to be able to offer crucial nuclear cardiology services in a manner that remains safe for patients and healthcare providers. Laboratories in most places with stable and low COVID case burden have now successfully opened up for elective testing and are using exercise stress testing (withheld during the acute phase of the pandemic) as needed.

These two documents by ASNC and SNMMI are not static and likely to be modified as new information continues to emerge. However, it is important to think that as the pandemic evolves with waxing and waning number of COVID-19 cases, the operations of nuclear cardiology laboratories will likely have to shift between performing only emergent cases where the test would yield an immediate and meaningful change in a patient's management and a more open model with doing nonemergent procedures as well.

Similarly to what Scrima et al. did, it will be important to continue monitoring, tracking and reporting the performance of our nuclear cardiology laboratories both in terms of proportion of abnormal cases and modalities utilized, as well as ensuring that the adherence to infection control protocols measures continues to prevent viral transmission to patients and workers.

ASNC has been impressively proactive in establishing new activities, and learning opportunities for trainees, technologists and practitioners. ${ }^{8}$ This pandemic, while ravaging as it may be, should make us stronger at the end by allowing us to operate together as members of the community, ensuring our safety and that of our patients, work with collaborators within our institutions and from around the world, learn to adapt to new constraints, re-examine our practice habits to increase efficiency in healthcare delivery, and strive to deliver excellent and equal clinical care to all.

\section{Disclosure}

Dr. Skali has no relevant disclosures to this manuscript.

\section{References}

1. WHO Director-General's opening remarks at the media briefing on COVID19. https://www.who.int/dg/speeches/detail/who-directorgeneral-s-opening-remarks-at-the-media-briefing-on-covid-19-11march-2020. Last accessed 09/06/2020

2. Krumholz HM. Where Have All the Heart Attacks Gone? New York Times April 06, 2020. https://www.nytimes.com/2020/04/06/ well/live/coronavirus-doctors-hospitals-emergency-care-heart-attac k-stroke.html. Last accessed 09/06/2020.

3. ASNC Webinar - COVID-19 Preparedness for Nuclear Cardiology Labs: Insights from the US, China and Singapore. https://www.a snc.org/ev_calendar_day.asp?date $=3 \% 2 \mathrm{~F} 24 \% 2 \mathrm{~F} 20 \&$ eventid $=98$. Last accessed 09/06/2020

4. Skali H, Murthy VL, Al-Mallah MH, et al. https://zenodo.org/rec ord/3738020. Accessed 09/06/2020

5. Skali H, Murthy VL, Al-Mallah MH, et al. Guidance and best practices for nuclear cardiology laboratories during the coronavirus disease 2019 (COVID-19) pandemic: An Information Statement from ASNC and SNMMI. J Nucl Cardiol 2020;27(3):1022-9. h ttps://doi.org/10.1007/s12350-020-02123-2.

6. ASNC Webinar - Restarting your Nuclear Lab Operations as the COVID-19 Pandemic Recedes. https://www.asnc.org/ev_calendar_ day. asp?date $=4 \% 2 \mathrm{~F} 30 \% 2 \mathrm{~F} 20 \&$ eventid $=105$. Last accessed $09 / 06 /$ 2020.

7. Skali H, Murthy VL, Paez D, et al. Guidance and best practices for reestablishment of non-emergent care in nuclear cardiology laboratories during the coronavirus disease 2019 (COVID-19) pandemic: An information statement from ASNC, IAEA, and SNMMI : Endorsed by the Infectious Diseases Society of America [published online ahead of print, 2020 Jul 24]. J Nucl Cardiol. 2020;1-8. https://doi.org/10.1007/s12350-020-02203-3

8. Dorbala S. ASNC's efforts during the COVID-19 pandemic. J Nucl Cardiol 2020;27:1084-5. https://doi.org/10.1007/s12350-020-0215 4-9.

Publisher's Note Springer Nature remains neutral with regard to jurisdictional claims in published maps and institutional affiliations. 